\title{
254. NOVEDADES FITOSOCIOLÓGICAS Y FLORÍSTICAS EN EL PARQUE NATURAL DE TEJEDA-ALMIJARA Y ALHAMA (MÁLAGA-GRANADA, ESPAÑA)
}

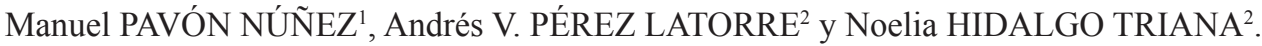

New records on the flora and vegetation of the Natural Park Sierras Tejeda-Almijara and Alhama (MalagaGranada, Spain).

Palabras clave. Euphrasia salisburguensis, Agrostis schleicheri, vegetación relicta, flora Alpino-Pirenaica, Andalucía.

Key words. Euphrasia salisburguensis, Agrostis schleicheri, relict vegetation, Alpine-Pyrenean flora, Andalusia.

Las Sierras de Tejeda, Almijara y Alhama, situadas al sur de Andalucía y entre las provincia de Málaga y Granada, están constituidas por una cordillera que rebasa ligeramente los 2000 metros de altitud. Estas sierras constituyen el límite más suroccidental de Europa para diversas comunidades de óptimo eurosiberiano y alpino (Pérez Latorre et al., 2004, 2015). Continuando con esta línea de investigación de vegetación relictual orófila y ombrófila-higrófila, hemos localizado e inventariado nuevas comunidades y sintaxones que pasamos a describir y/o comentar. La nomenclatura sigue a Blanca et al. (2011) excepto para Solidago virgaurea subsp. minuta (L.) Arcangeli (identificada según Bolòs et al., 1990). La metodología fitosociológica utilizada es la de Braun-Blanquet (1979) y la sintaxonomía y bioindicadores de Rivas Martínez (2011).

\section{Comunidad con Euphrasia salisburgensis y Agrostis schleicheri [Tabla 1] \\ Euphrasia salisburgensis Funk. es un} microterófito ocasionalmente hemiparásito propio de pastizales calcícolas quionófilos subalpinos-alpinos o también de zonas rocosas expuestas en los pisos orotemplado y supratemplado superior de las altas montañas eurosiberianas europeas (KoebresioSeslerietea) (Rivas-Martínez et al., 2011). La clase Koebresio-Seslerietea Br.-B1. 1948 [syn.: Festuco-Seslerietea, Elyno-Seslerietea] alcanza de forma relicta y finícola algunas montañas mediterráneas ibéricas, haciéndose muy rara hacia el sur a la vez que Erinacetalia y Festuco-Brometea cobran importancia en estas comunidades (Rivas Goday \& Borja, 1961). En Sierra Tejeda, la comunidad con Euphrasia salisburgensis se ubica en microcenotopos inclinados, con suelos arenosos dolomíticos, temporalmente húmedos, y bajo la protección de potentes farallones calizos orientados al norte. El hábitat se encuentra en el límite entre los pisos supra y oromediterráneo húmedo (Pérez Latorre et al., 2004). Contacta con la asociación tejedense brio-cormofítica de paredes calizas rezumantes Southbyo tophaceae-Pinguiculetum dertosensis Asensi \& Díez Garretas 2002.

Euphrasia salisburgensis junto con otros taxones presentes en la zona (Botrychium lunaria (L.) Swartz, Gymnocarpium robertianum (Hoffm.) Newman, Gymnadenia conopsea (L.) R. Br., Sorbus latifolia (Lam.) Pers. ó Achnatherum calamagrostis (L.) P. Beauv., entre otros), hacen de la vertiente norte de Sierra Tejeda uno de los más importantes reductos 
Tabla 1

1. Comunidad con Euphrasia salisburguensis y Agrostis schleicheri

(Asplenietea, Violo biflorae-Cystopteridetalia, Violo biflorae-Cystopteridion alpinae)

(Koebresio myosuroidis-Seslerietea caeruleae)

INVENTARIO N

Orientación

Inclinación $\left({ }^{\circ}\right)$

Área $\left(\mathrm{m}^{2}\right)$

Litología

Altitud (m)

Altura de la veg. (cm)

Cobertura

$\begin{array}{ccccc}1 & 2 & 3 & 4 & 5 \\ \mathrm{~N} & \mathrm{~N} & \mathrm{~N} & \mathrm{~N} & \mathrm{~N} \\ 50 & 0 & 30 & 40 & 10 \\ 1 & 0,25 & 0,25 & 0,25 & 0,50 \\ \text { Dol } & \text { Dol } & \text { Dol } & \text { Dol } & \text { Dol } \\ 1770 & 1790 & 1800 & 1800 & 1800 \\ 15 & 10 & 3 & 3 & 10 \\ 60 & 40 & 80 & 70 & 90\end{array}$

Violo biflorae-Cystopteridetalia alpinae

Agrostis schleicheri

1

3

2

Koebresio-Seslerietea caeruleae

Euphrasia salisburgensis

2

Adiantetea y Pinguiculion longifoliae

Pinguicula dertosensis

$(+)$

2

1

\section{Caricetea curvulae}

Solidago virgaurea subsp. minuta

Potentilletalia caulescentis y Saxifragion camposii

Potentilla caulescens

Hieracium texedense

Campanula rotundifolia subsp. hispanica

Jasione foliosa subsp. minuta

Sinusia briofítica: Bryum sp., Distichum capillaceum,

Didymodon acutus, Plagiochasma rupestre

\section{Compañeras}

Ranunculus paludosus

Trisetum velutinum

Brassica repanda subsp. latisiliqua

Centaurium barrelieri

Sedum album

$\begin{array}{lllll}1 & . & 1 & \cdot & + \\ 3 & \cdot & \cdot & + & + \\ + & \cdot & \cdot & + & . \\ + & \cdot & \cdot & \cdot & \cdot \\ \cdot & \cdot & + & \cdot & .\end{array}$

Localidades. 1, 2, 3, 4 y 5: Granada. Alhama de Granada. Parque Natural Sierras de Tejeda, Almijara y Alhama. Salto del Caballo. 30SVF0884. Cenotopos: 1. Concavidad con Juniperus communis. 2. Repisa tobácea horizontal. 3, 4 y 5. Césped almohadillado sobre suelo arenoso dolomítico temporalmente húmedo. Dol: dolomías. 
de flora relicta eurosiberiana y boreo-alpina en las montañas calizo-dolomíticas del sur de España, que con mayor o menor intensidad se vieron afectadas por el glaciarismo cuaternario reciente (Cabezudo \& Pérez Latorre, 2006; Pérez Latorre et al., 2014, 2015).

La primera cita de Euphrasia salisburgensis corresponde a Blanca et al. en 1983 en la localidad Salto del Caballo, Sierra Tejeda (GDAC 29238-1G) (Pérez-García \& Mota, 2009). Treinta y dos años después, hemos localizado y contabilizado tres poblaciones en la misma localidad; la primera de unos 100 individuos bajo un potente paredón calizo extraplomado, la segunda población de unos 25 individuos en una grieta-repisa horizontal con protosuelo húmedo y finalmente una tercera población de 3 individuos en un pastizal de base de cantil [MGC 81720: Granada, Alhama de Granada, Sierra Tejeda. Salto del Caballo. 1760 m. 30SVF0884, 10-8-2015. Leg. A. V. Pérez Latorre \& M. Pavón Núñez]. Estos datos contribuyen a valorar la situación actual del status de conservación de esta especie en Andalucía y las medidas de protección necesarias en la zona.

En la comunidad, es importante en presencia y cobertura Agrostis schleicheri Jordan \& Verlot [MGC 81722-81721: Granada, Alhama de Granada, Sierra Tejeda, Salto del Caballo. 1760 m. 30SVF0884, 10-8-2015. Leg. A. V. Pérez Latorre \& M. Pavón Núñez]. Se trata de la primera cita de este taxón para el Parque Natural, para la provincia de Granada y para el extremo sur de la Península Ibérica (Romero García et al., 1988; Cabezudo et al., 2005). En toda la mitad sur de la Península, tan sólo existe una población más en la Sierra de Cazorla (Blanca et al., 2011; Gómez Mercado, 2011). $\mathrm{Su}$ escasez en Andalucía podría determinar su grado de amenaza según UICN, que sería necesario evaluar.

La comunidad se considera un fragmento relictual empobrecido con una agrupación de especies procedentes de diversas clases fitosociológicas de apetencias orófilas e higrófilas boreo-alpinas y templadas (Rivas Martínez, 2011) y por lo tanto, hoy día las consideramos relictuales en la zona:

\section{a. Koebresio myosuroidis-Seslerietea} caeruleae: comunidades cespitosas y camefítico-pulviniformes, orófilas, basófilas, sobre roquedos o coluvios calcáreos en los pisos supra-orotemplado húmedoultrahiperhúmedo orocantábricos, pirenaicos, alpinos y apenino-balcánicos.

b. Violo biflorae-Cystopteridion alpinae: comunidades rupícolas, esciófilas, quionófilas y mesohigrofíticas, alpino-pirenaicoorocantábricas, oro-criorotempladas, húmedo-ultrahiperhúmedas.

c. Caricetea curvulae: comunidades cespitosas orófilas, quionófilas, silicícolas y geliremovidas, oro-criorotempladas, húmedo-hiperhúmedas, alpino-pirenaicas, orocantábricas y carpáticas.

d. Pinguiculion longifoliae: comunidades de roquedos calcáreos rezumantes, ricas en especies insectívoras, mesosupramediterráneas seco-húmedo y supratemplado húmedo-hiperhúmedo submediterráneo.

Además, existe un componente florístico de Potentilletalia caulescentis que proviene de la asociación Hieracio texedensis-Jasionetum minutae Pérez Latorre \& Cabezudo 2004, propia de roquedos supra-oromediterráneos de la zona de estudio (Pérez Latorre et al., 2004).

\section{Comunidad con Populus tremula [Tabla 2]}

Las formaciones vegetales donde aparece el álamo temblón (Populus tremula L.) son propias de las montañas de la mitad septentrional de la Península Ibérica, bajo el dominio del macrobioclima templado (Ruiz de la Torre, 2006). A medida que nos desplazamos hacia el sur, la presencia de esta especie disminuye paulatinamente, quedando acantonada 
Tabla 2

\section{Comunidad con Populus tremula \\ (Querco-Fagetea, \\ Betulo pendulae-Populetalia tremulae)}

\begin{tabular}{lcc}
\hline INVENTARIO N & 1 & 2 \\
Orientación & $\mathrm{NE}$ & $\mathrm{SE}$ \\
Cobertura $(\%)$ & 90 & 95 \\
Inclinación $\left({ }^{\circ}\right)$ & 0 & 0 \\
Área $\left(\mathrm{m}^{2}\right)$ & 100 & 100 \\
Litología & Dol & Dol \\
Altitud (m) & 1100 & 1105 \\
Altura de la veg. $(\mathrm{m})$ & 20 & 25
\end{tabular}

Betulo pendulae-Populetalia tremulae

Populus tremula

Populus $x$ canescens

1

3

3

2

Salici-Populetea y Populetalia albae

Populus alba

Populus nigra

2

$+$

Querco-Fagetea

Hedera hibernica

Daphne laureola

Helleborus foetidus

Sorbus aria

Viola riviniana

Primula veris

Acer granatense

\section{Rhamno cathartici-Prunetea spinosae}

Rubus ulmifolius

Berberis hispanica

Ononis aragonensis

Rosa pouzinii

$\begin{array}{ll}1 & 1 \\ + & + \\ + & + \\ + & + \\ + & + \\ \cdot & 1 \\ . & +\end{array}$

Compañeras

Tamus communis

Carex mairii

Brachypodium sylvaticum

Oryzopsis paradoxa

Rubia peregrina

Asparagus acutifolius

Euphorbia characias

2
+
+
+

Otras compañeras. En 1: Pteridium aquilinum 4, Clematis flammula +, Scirpus holoschoenus 1, Daphne gnidium +. En 2: Molinia caerulea 1, Ruscus aculeatus + .

Localidades. 1 y 2: Granada. Alhama de Granada. Parque Natural Sierras de Tejeda, Almijara y Alhama. Arroyo del Cerezal. 30SVF1083. Dol: dolomías. en zonas microclimáticas como laderas umbrosas o arroyos de curso permanente de las altas montañas meridionales. En Andalucía, sus poblaciones quedan confinadas a las montañas béticas orientales, habiéndose citado formaciones generalmente de pocos individuos en Sierra Nevada y Sierra Harana (Morales et al., 2001), siendo la más numerosa la de Sierra de Baza, al parecer con algunos centenares de individuos (VV. AA., 1998).

En Sierra Tejeda, la comunidad con Populus tremula, no detectada hasta ahora (Pérez Latorre et al., 2004), aparece puntualmente y restringida a cursos de aguas permanentes con luvisoles formados a partir de las dolomías masivas circundantes, en el piso mesomediterráneo subhúmedo del distrito Tejedense, constituyendo posiblemente la población más meridional europea (Pérez Latorre et al., 2014). Dicha comunidad está representada esencialmente por Populus tremula y Populus alba L., estando presente también en el microcenotopo el híbrido de ambas especies Populus $x$ canescens (Aiton) Sm. Además, coexisten con otros caducifolios de Querco-Fagetea tales como Sorbus aria (L.) Crantz o Acer opalus Mill. subsp. granatense (Boiss.) Font Quer \& Rothm., dando lugar a formaciones tipo galería, cuya orla espinosa es un zarzal de Rubus ulmifolius Schott acompañado ocasionalmente por rosas (Rosa pouzinii Tratt.) y agracejos (Berberis hispanica Boiss. \& Reut.). Actualmente, la dinámica de Populus tremula en estos bosquetes de ribera podría ser de regresión. Se han localizado sólo cinco individuos, cuatro adultos y un ejemplar juvenil en las dos localidades inventariadas (supone un aumento de tres individuos respecto a los datos de Pérez Latorre et al., 2014) frente a la pujanza de Populus alba y sus híbridos Populus $x$ canescens. Posiblemente los bosquetes con $P$. tremula estuviesen más extendidos en un pasado más frío (Pérez Latorre et al., 2015) y representen fragmentos de Betulo-Populetalia tremulae excepcionales 
en Sierra Tejeda, muy escasos en la provincia corológica Bética (Pavón \& Pérez Latorre, 2010) y los más meridionales de Europa (Rivas Martínez, 2011).

\section{Daphno latifoliae-Aceretum granatensis pistacietosum terebinthi Gómez Mercado} \& Valle 1990

La cornicabra (Pistacia terebinthus L.) es un microfanerófito caducifolio de amplia representación en el piso mesomediterráneo de las montañas calizas de la provincia Bética (Blanca et al., 2011). Su mayor resistencia a la xericidad estival que otras especies caducifolias (Valle et al., 1989), por un lado, y su fisionomía de taxón deciduo de invierno por otro, determina una polivalencia fitocenológica y sintaxonómica un tanto singular (Pavón et al., 2013). Así en los cornicabrales béticos actúa como especie directriz en la asociación Phillyreo latifoliae-Pistacietum terebinthi, propia de biotopos kársticos perteneciente a la clase Pistacio-Rhamnetalia (Pavón et al., 2013). No obstante, esta misma especie define la faciación térmofila de los quejigaresacerales béticos en el piso mesomediterráneo subhúmedo-húmedo Daphno latifoliaeAceretum granatensis pistacietosum terebinthi incluida en el orden Aceri-Quercion fagineae de la clase Quercetalia pubescentis (Gómez Mercado \& Valle, 1990). De esta subasociación hay que precisar, sin embargo, que solo presenta estrato arbóreo dominante de Q. faginea Lam., desapareciendo no solo $A$. granatense sino todo su cortejo florístico nemoral (ver tabla 3 invs. 9-14 en Gómez Mercado \& F. Valle, 1990: 247 y tabla 84 invs. 9-14 en Gómez Mercado, 2011: 178-179), por lo que quizá un estudio más profundo de esta subass. lleve a la descripción de un quejigar mesomediterráneo independiente o relacionado con Viburno tini-Quercetum fagineae Torres \& Cano in Cano, Pinto-Gómes, F. Valle, J. Torres, García- Fuentes, Salazar, Melendo \& Mendes 2003 de areal también subbético.
Destacamos como novedad sincorológica la presencia testimonial de esta subasociación no detectada hasta ahora (Pérez Latorre et al., 2004) en el sector Almijaro-Granatense y en concreto en Sierra Tejeda. Damos a conocer su existencia con un inventario realizado en Arroyo de las Piletas (Alhama de Granada, Granada), 30SVF1086, fondo de vaguada:

Altitud: $1130 \mathrm{~m}$. Inclinación: $0^{\circ}$. Litología: dolomías. Orientación: E-SE, Cobertura: 90\%, Altura vegetación: $6 \mathrm{~m}$. Características de subasociación: Acer granatense 4, Pistacia terebinthus 1, Daphne laureola subsp. latifolia + . Características de unidades superiores: Quercus faginea +, Heleborus foetidus +, Sorbus aria +. Compañeras: Amelanchier ovalis +, Berberis hispanica 1, Rubus ulmifolius 2, Ononis aragonensis 2, Rosa pouzinii 1, Tamus communis +, Brachypodium sylvaticum 1, Piptatherum paradoxum 2, Rubia peregrina 1, Arrhenatherum bulbosum +, Pinus sylvestris +, Quercus rotundifolia + , Juniperus phoenicea + , Celtis australis $(+)$.

\section{Elymo hispanici-Brachypodietum sylvatici}

Gómez-Mercado \& F. Valle 1991.

Asociación graminoide y hemicriptofítica de elevada talla, de suelos profundos y frescos a la sombra de bosques y espinares caducifolios, supra y mesomediterráneos del sector Subbético (Gómez Mercado, 2011). Hemos detectado su presencia en otros dos sectores fitogeográficos de la provincia Bética, ampliándose por tanto su área de distribución a las Sierras del Torcal y Camarolos (sector Antequerano) y Sierra de Tejeda (sector Almijaro-Granatense). En estos dos sectores constituye la orla herbácea vivaz de formaciones boscosas caducifolias climácicas tales como acerales béticos (Daphno latifoliaeAceretum granatensis Rivas-Martínez 1965) y quejigales mixtos torcalenses (Vinco difformisQuercetum fagineae aceretosum monspessulani Pavón, Hidalgo \& Pérez Latorre in Pavón \& Pérez Latorre 2013). Presentamos esta novedad sincorológica mediante un inventario realizado 
en el dominio del aceral bético Daphno latifoliae-Aceretum granatensis en suelo profundo con hojarasca y humus, en el piso supramediterráneo húmedo de Sierra Tejeda, Las Llanadas, cabecera del Arroyo de la Cueva de la Parra (Alhama de Granada, Granada), 30S VF1184, fondo de vaguada:

Altitud: $1358 \mathrm{~m}$. Inclinación: $15^{\circ}$. Litología: dolomías. Orientación: NW. Cobertura: 75\%. Altura de la vegetación: 1,2 m. Características de asociación: Brachypodium sylvaticum 2, Elymus hispanicus 1, Piptatherum paradoxum 1. Compañeras: Bunium macuca+, Clinopodium vulgare 1, Polygonatum odoratum + , Luzula baetica +, Lapsana communis 1, Pteridium aquilinum 1, Helleborus foetidus +, Cerastium boissieri 1, Primula veris +, Geranium purpureum 1, Daphne laureola + , Paeonia coriacea + , Rubia peregrina + , Tamus communis + .

\section{Esquema sintaxonómico}

KOBRESIO MYOSUROIDIS-SESLERIETEA CAERULEA Br.-B1. 1948 em. Ohba / ASPLENIETEA TRICHOMANIS (Br.-Bl. in Meier \& Br.-B1. 1934) Oberdorfer 1977

+ Violo biflorae-Cystopteridetalia alpinae F. Casas 1970

* Violo biflorae-Cystopteridion alpinae F. Casas 1970

1. Comunidad con Euphrasia salisburgensis y Agrostis schleicheri [Césped graminoide briofítico con microterófitos, semihigrofítico, supra-oromediterráneo húmedo, de base de cantiles calizos umbríos]

QUERCO-FAGETEA Br.-Bl. \& Vlieger in Vlieger 1937

+ Betulo pendulae-Populetalia tremulae Rivas-Martínez \& Costa 2002

2. Comunidad con Populus tremula [Chopera con álamo temblón, relicta, mesomediterránea, edafohigrófila, almijaro-granatense]
+ Quercetalia pubescenti-petraeae Klika 1933

* Aceri granatensis-Quercion fagineae (Rivas Goday, Rigual \& Rivas Martínez in Rivas Goday \& al. 1960) Rivas Martínez 1987

3. Daphno latifoliae-Aceretum granatensis pistacietosum terebinthi Gómez-Mercado \& Valle 1990 [Aceral bético termófilo, mesomediterráneo, basófilo, con cornicabras]

TRIFOLIO MEDII-GERANIETEA SANGUINEI Müller 1962

+ Origanetalia vulgaris Müller 1962

* Origanion virentis Rivas-Martínez \& O. Bolòs in Rivas-Martínez, T.E. Díaz, F. Prieto, Loidi \& Penas 1984

** Origanienion virentis (Rivas-Martínez \& O. Bolòs in Rivas-Martínez, T.E. Díaz, F. Prieto, Loidi \& Penas 1984) Capelo 1996

4. Elymo hispanici-Brachypodietum sylvatici Gómez-Mercado \& F. Valle 1991 [Herbazal graminoide esciohumícola, supramesomediterráneo, basófilo, de bosques caducifolios béticos]

AGRADECIMIENTOS. Al Dr. Juan Guerra (UM) por la identificación del material briofítico. A la Dra. M. T. Romero (UGR) por la revisión del material de Agrostis.

\section{BIBLIOGRAFÍA}

BLANCA, G., B. CABEZUDO, M. CUETO, C. FERNÁNDEZ LÓPEZ, C. MORALES TORRES y C. SALAZAR. (eds.) -2011-. Flora Vascular de Andalucía Oriental. (2 $2^{\mathrm{a}}$ edición corregida y aumentada). Consejería de Medio Ambiente. Junta de Andalucía. Sevilla. 1751 pp.

BRAUN-BLANQUET, J. -1979- Fitosociología, Ed. Blume, Madrid. $820 \mathrm{pp}$.

BOLÒS, O., J. VIGO, R.M. MASALLES y J.M. NINOT -1990- Flora Manual dels Països Catalans. Pòrtic. Barcelona. 1247 pp.

CABEZUDO, B., A. V. PÉREZ LATORRE, D. NAVAS, O. GAVIRA y G. CABALLERO -2004- Contribución al conocimiento de la 
flora del Parque Natural de las Sierras Tejeda y Almijara (Málaga-Granada, España). Acta Bot. Malacitana 30: 55-110.

CABEZUDO B. y A. V. PÉREZ LATORRE2006- Datos sobre la vegetación relicta con Gymnocarpium robertianum (Hoffh.) Newman en el sur de la Península Ibérica. Acta Bot. Malacitana 31: 174-177.

GÓMEZ MERCADO, F. -2011-. Vegetación y flora de Sierra de Cazorla. Guineana 17: 5-481. Universidad del País Vasco.

GÓMEZ MERCADO, F. y F. VALLE -1990- Notas fitosociológicas sobre las comunidades arbóreas de las Sierras de Cazorla y Segura. Acta Bot. Malacitana 15: 239-246.

MORALES, C., C. QUESADA y L. BAENA.-2001Guías de la naturaleza. Árboles y arbustos. (Los libros de la estrella; 6. Ciencia y Medio Ambiente). Diputación Provincial. Granada. $217 \mathrm{pp}$.

PAVÓN NÚÑEZ, M. y A. V. PÉREZ LATORRE -2010- Sobre las avellanedas subbéticas con Ulmus glabra Huds. en las Sierras de CazorlaSegura-Alcaráz, Jaén-Albacete (España). Lagascalia 30: 393-405.

PAVÓN NÚÑEZ, M., N. HIDALGO TRIANA y A. V. PÉREZ LATORRE -2013- Aportaciones al conocimiento de las comunidades de Pistacia terenbinthus L. y de Acer monspessulanum L. en el Sur de la Península Ibérica. Lagascalia 33: 299-311.

PÉREZ-GARCÍA, J. y J. F. MOTA -2009- Sobre el género Euphrasia L. (Scrophulariaceae) en el sur de la Península Ibérica. Acta Bot. Malacitana 34: 287-289.

PÉREZ LATORRE, A. V., D. NAVAS, O. GAVIRA, G. CABALLERO y B. CABEZUDO -2004Vegetación del Parque Natural de las Sierras Tejeda, Almijara y Alhama (Málaga-Granada, España). Acta Bot. Malacitana 29: 117-190.

PÉREZ LATORRE, A. V., M. PAVÓN NÚÑEZ, N. HIDALGO TRIANA y B. CABEZUDO -2014- Caducifolios arbóreos relícticos en zonas mediterráneas (Andalucía, España): tipos funcionales, fitocenología y conservación. In: Cámara, R., B. Rodríguez \& J. L. Muriel (eds.). Biogeografía de Sistemas Litorales. Dinámica y Conservación. pp. 381-385. Sevilla.

PÉREZ LATORRE, A. V., M. PAVÓN NÚÑEZ y N. HIDALGO TRIANA, -2015-Vegetación higrófila de alta montaña con Sesleria argentea (Savi) Savi (Poaceae) en el sur de la Península Ibérica: Sierra Tejeda (Granada, Málaga). Anales de Biología 37: 11-24.

RIVAS GODAY, S. y J., BORJA CARBONELL -1961- Estudio de vegetación y flórula del Macizo de Gúdar y Javalambre. Anales del Instituto Botánico Cavanilles 19: 3-543.

RIVAS-MARTÍNEZ, S. -2011- Mapa de series, geoseries y geopermaseries de vegetación de España. Memoria del Mapa de Vegetación Potencial de España. Partes I y II. Itinera Geobot. 18: 5-424.

ROMERO GARCÍA, A. T., G. BLANCA LÓPEZ y C. MORALES TORRES -1988- Revisión del género Agrostis L. (Poaceae) en la Península Ibérca. Ruizia 7: 5-160.

RUIZ DE LA TORRE, J. - 2006- Flora Mayor. ICONA (Organismo Autónomo Parques Nacionales). Madrid. 1756 pp.

VALLE, F., F. GÓMEZ MERCADO, J. MOTA y C. DÍAZ DE LA GUARDIA -1989- Parque Natural de Cazorla, Segura y Las Villas. Guia botánicoecológica. Editorial Rueda. Madrid. 354 pp.

VV. AA. -1998- Guía para conocer y visitar el Parque Natural Sierra de Baza. Baza. 392 pp.

Dirección de los autores: 'IES Playamar, Ronda Alta Benyamina, 29620, Torremolinos (Málaga). ${ }^{2}$ Dpto. de Biología Vegetal. Facultad de Ciencias. Universidad de Málaga. 29071, Málaga. *Autor para correspondencia: manuelp1764@gmail.com 